\title{
INESTABILIDAD LABORAL Y COMPETITIVIDAD
}

Norberto E. Garcia (*)

IEP, Lima

Palabras clave: competitividad, America Latina, reformas laborales, inestabilidad laboral.

RESUMEN:

En las dos últimas décadas pasadas - 1980's y 1990's - , en buena parte de los paises de América Latina y el Caribe se introdujeron reformas laborales para flexibilizar el mercado laboral - Argentina, Brasil, Chile, Ecuador, Perü, Colombia, Panamá, entre otros - . En particular, siguiendo la experiencia de España de 1983-97, la mayoría de esas reformas promovieron la introducción de múltiples contratos laborales y redujeron los costos de indemnización por despido. La introducción de los múltiples contratos o contratos flexibles como se les denominó en América Latina se efectuó con dos argumentos esenciales: que promoverían el aumento del empleo al disminuir el costo de contratación; y que contribuirían al aumento de la competitividad de las empresas, becho fundamental en el proceso

- Investigador Asociado del IEP, Lima, Investigador Líder de la Red de Políticas de Empleo, Consorcio de Investigaciones Económicas y Sociales (CIES) del Perú, y consultor internacional en temas de economía laboral. 
de apertura y reinserción en la economia internacional que estos países habian comenzado a transitar.

Key words: competitiveness, Latin America, labour reformations, labour uncertainly.

ABSTRACT:

In the two last decades-1980's and 1990's -, most of the Latin American and Caribbean countries, labour reformations were introduced to make flexible the labour market-Argentina, Brazil, Chile, Ecuador, Peru, Colombia, Panama, among otber -. In particular, following the Spanish experience during 1983-97. most of those reformations promoted the introduction of different labour contracts and reduced the costs for dimisal. The introduction of these contracts or flexible contracts, as they are denominated in Latin America, was made because of two essential arguments: to increase the employment when the contracting cost decrese; and to contribute to the increase of the competitiveness of the companies, fundamental fact on the opening process and reinsertion in the international economy that these countries are focusing.

\section{INTRODUCCION}

En las dos décadas pasadas 1980 y 1990, en buena parte de los países de América Latina y el Caribe se introdujeron reformas laborales para flexibilizar el mercado laboral -Argentina, Brasil, Chile, Ecuador, Perú, Colombia, Panamá, entre otros-. En particular, siguiendo la experiencia de España de 1983-97, la mayoría de esas reformas promovieron la introducción de múltiples contratos laborales y redujeron los costos de indemnización por despido. La introducción de los múltiples contratos o contratos flexibles como se los denominó en América Latina- o estímulos a la flexibilidad de "entrada" al mercado laboral -, se efectúo con dos argumentos esenciales: i) que promovería el aumento del empleo al disminuir el costo de contratación; y ii) que contribuiría al aumento de la competitividad de las empresas, hecho fundamental en el proceso de apertura y reinserción en la economía internacional que estos países habían comenzado a transitar. Lo cuestionado fue la estabilidad laboral, tanto por razones de los mayores costos como por su efecto nocivo sobre la competitividad de las empresas.

No obstante, la evidencia empírica que comienza a aflorar, una o dos décadás después de puestas en práctica las reformas laborales, sugiere que la óptica inicial de las mismas es dudosa, y que parece imprescindible analizar con más atención làs implicaciones del enfoque inicial

No deja de llamar la atención que en los tres únicos países exitosos en materia de apertura, reformas y restructuración productiva para generar un segmento competitivo. 
- Chile, México y Costa Rica - las reformas laborales adquirieron matices diferentes. Así, en Chile la profunda reforma laboral de 1975 fue significativamente atemperada en 1990, con una nueva reforma laboral aprobada en el primer Gobierno democrático que asume la administración del país después del interregno 1973-89. La reforma de 1990 suaviza la institucionalidad anterior, justamente en el tema de múltiples contratos y despido. Costa Rica y México son ejemplos aún más claros: ninguno de los dos paises introduce reformas laborales en la dirección de profundizar las reglas del juego en materia múltiples contratos y despido.

Los cambios institucionales en España registrados entre 1997 y el presente en los dos temas antes citados - múltiples contratos y despido -, no fueron absorbidos en los países de América Latina y el Caribe con la misma receptividad que los adoptados por España en 1983. Esto hace que la discusión de los temas expuestos sea más relevante aún.

Este trabajo se concentra en el tema de los efectos de múltiples contratos en el Perú. Se ha escogido esta experiencia, porque es uno de los países que adopta la reforma laboral más profunda en el ámbito citado. En parte se presenta evidencia de esta experiencia, pero también se levantan hipótesis que deberán ser todavía verificadas empíricamente.

\section{Costos e InESTABILIDAD LABoral}

La introducción de múltiples contratos en la experiencia Peruana a partir de la reforma laboral de 1991, se inscribe en una tendencia hacia el descenso de la estabilidad laboral verificada de hecho entre 1970 y el presente. Dicho de otro modo, la tendencia subyacente en los mercados laborales era ya hacia una menor estabilidad contractual, a través de la evasión de las disposiciones laborales vigentes. Pero la apertura de la economía Peruana a principios de los 90 coloca una fuerte y abrupta presión sobre la capacidad competitiva de las empresas, que es transmitida hacia los mercados laborales.

El argumento central era la necesidad de reducir los costos de la mano de obra para poder competir, para lo cual la disponibilidad de contratos temporales, eventuales, de locación de servicios y otras formas contractuales, aparecía como una solución ya que el retraso de la tasa de cambio real, y una tasa de interés muy elevada, no dejaban mucho margen a las empresas para actuar sobre otros factores que inciden en los costos de sus insumos.

Durante los 90 se redujo efectivamente el costo laboral promedio por unidad de trabajo, por desplazamiento de la ocupación asalariada hacia contratos más "baratos".

Así, para tomar como referencia la información de la Industria Manufacturera, un sector que suele comportarse cercano al promedio de los sectores transables - expuestos a la competitividad -, el costo laboral medio de un contrato permanente ascendía a mediados de los 90 a us. 2.1 dls la hora, mientras que el de un contrato "flexible" 
llegaba a us. $1.37 \mathrm{dls}$ la hora, y en el caso de un asalariado sin contrato o contratado en negro, alcanzaba us. 1.1 dls la hora (Martínez, D. y Tokman, V.)

El Cuadro 1 sugiere con claridad que en los 90's la ocupación asalariada se desplazó hacia los contratos más "baratos", y por esta vía se redujo el costo laboral promedio.

Llama la atención que el grueso de este desplazamiento -como se desprende del Cuadro 1 -, haya tenido lugar a través de los contratos en negro o personal sin contratos -, definidos estadísticamente como aquellos que declaraban no tener contrato laboral y no cotizar ni seguro de salud ni seguro de pensiones o jubilaciones. En la discusión previa a la reforma en torno a la flexibilización del mercado laboral, nunca emergió este tema, que en cierto modo fue un resultado no esperado de este proceso - aún cuando las tendencias subyacentes en materia de crecimiento de la inestabilidad laboral ya citadas, deberían haber servido de advertencia al respecto-.

Una vía adicional a través de la cual se buscó el descenso de los costos laborales fue la reducción de los llamados sobre costos laborales no salariales.

\section{Cuadro 1.}

Perú. Empleo Asalariado Privado por Modalidad Contractual. 1991-2000

\begin{tabular}{|lccc|}
\hline \multicolumn{4}{c|}{ (porcentajes) } \\
\hline & Lima 1991 & Lima 2000 & Perú \\
\hline Urbano 2000 & & & \\
Con contrato estable & 41.0 & 24.8 & 21.3 \\
Con contrato no estable & 25.8 & 29.8 & 31.9 \\
Sin Contrato (1) & 33.2 & 45.4 & 46.8 \\
Total & 100 & 100 & 100 \\
\hline
\end{tabular}

Fuente: Chacaltana, J. y García, $N$ (2001) basándose en información de Encuestas Nacionales de Hogares, INEI, Lima, Perú.

(1) Asalariados privados que en la encuesta afirman estar trabajando sin contrato y que simultaneamente, no registran cotización ni al seguro de salud ni al de pensiones.

Esto no generó cambios dramáticos en materia de costos laborales por unidad. producida, pero atendió a las presiones empresariales que reclamaban su reduceión.

El Cuadro 1 también sugiere que afines de los 90, como consecuéncia de los: cambios institucionales, sólo un 21 por ciento de los asalariados tenían un contrato: laboral permanente o estable, mientras que un 79 por ciento estaba ocupado; 
Cuadro 2.

Empleo Asalariado según Contratos y Tamaño de Establecimiento. 2000

\begin{tabular}{|lrrr|}
\hline \multicolumn{4}{c|}{ (porcentajes) } \\
\hline & Estable & No Estable & Sin Contrato \\
\hline < 10 ocupados & 24.4 & 19.3 & 72.6 \\
10 a 49 ocupados & 18.3 & 27.2 & 20.1 \\
50 a 99 ocupados & 9.5 & 11.4 & 2.6 \\
100 y más ocupados & 47.8 & 42.1 & 4.6 \\
Total & 100 & 100 & 100 \\
\hline
\end{tabular}

Fuente: Chacaltana, J. y Garcia, N. (2001), basándose en información de las Encuestas Nacionales de Hogares, LNEI, Lima.

principalmente, con contratos en negro ( $47 \%$ ) y en menor medida con contratos flexibles (32\%). Respécto a estos últimos, un $90 \%$ de los ocupados con este tipo de contratos, correspondían al uso de dos de las figuras contractuales: los contratados en forma eventual o temporal, y los ocupados a través de un contrato de locación de servicios. La legislación que emergió de la reforma laboral, establecía un máximo de 5 años acumulados para el uso del primer tipo de contratos y no establecía límite alguno para el segundo. De esta manera, aprovechando la laxitud de las normas, en la práctica muchas empresas utilizaron estas figuras contractuales no para satisfacer la legítima finalidad de contratos eventuales o de locación de obra, sino para trasladar un porcentaje de los empleós permanentes hacia contratos temporarios y lograr la permanencia por constantes renovaciones sujetas a la aprobación de la empresa.

No obstante, como emerge del Cuadro 1, la principal fuente de flexibilidad verificada en los 90 es el impresionante aumento en la proporción de asalariados privados sin contrato o en negro, que a fines de los 90, como fue expuesto, alcanzaba el 47 por ciento. Parece evidente que al calor de las grandes discusiones para legitimar un mercado laboral más flexible, lo que sucedió en la práctica es una brusca acentuación de las tendencias de hecho hacia la paulatina desregulación del mercado laboral a través de una significativa ampliación de la evasión, poco riesgosa en los 90 porque en esa década el Ministerio de Trabajo redujo a un mínimo su capacidad de fiscalización.

El Cuadro 2 parece confirmar esta hipótesis, ya que es en el tramo de pequeña y micro empresa donde se registra la mayor proporción de contratos en negro o no registrados, mientras la mayor ponderación de contratos flexibles se encuentra en la mediana y gran empresa. Dado que la evasión a todo tipo de normas incluyendo las laborales es mayor en el segmento de la pequeña y microempresa, es esperable un resultado como el señalado por el Cuadro 2. Además, conviene recordar que en los 90, el crecimiento del empleo 
verificado en el Perú, tuvo lugar en autoempleo (cuenta propia) y en empleo en micro y pequeña empresa, - lo que tendió a incrementar la proporción de ocupados sin contrato o no registrados, por el alza en la ponderación del empleo en pequeña y micro empresa en el empleo asalariado total.

Las consecuencias sociales de este fuerte aumento de la inestabilidad del empleo son conocidas: elevación de la precariedad laboral, fuerte contracción de la sindicalización, reducción del nivel de vida de los afectados, incertidumbre hacia el futuro, efectos negativos sobre las cotizaciones para la seguridad social, etc. Pero este tremendo precio no parece haber sido el único que debió pagarse para alcanzar una controvertida competitividad en el mercado laboral. Existe evidencia que también se pago un precio en términos de la competitividad misma. Esto último se desarrolla en la sección siguiente.

\section{Costos y Productividad}

En la sección previa se hablaba implícitamente de costos laborales por unidad de trabajo. No obstante, lo relevante para fines de competitividad son los costos laborales por unidad de producto - y en general, todos los costos por unidad de producto-. El análisis económico ha establecido que los costos por unidad de producto pueden plantearse como la relación entre los costos por unidad de insumo y la productividad de dicho insumo. En el caso de la mano de obra, estarían dados por la relación entre el costo laboral por hora y la productividad por hora (Chacaltana, J. y García, N.) Esta aclaración es importante porque sin ella no se entendería por qué países con bajos costos laborales por hora - por ejemplo el Perú, con un costo laboral en la industria de us. 2.1 dls por hora son menos competitivos que experiencias en las que los costos laborales son diez veces más altas - por ejemplo Alemania, con un costo laboral en la industria de us. 28.- dls por hora.

Por consiguiente, para fines de mejorar la competitividad, la reducción del costo laboral por unidad producida puede lograrse incrementando la productividad o bajando el costo laboral por unidad de trabajo - y esta última fue la senda escogida por el Perú y otros países de América. Al escoger esta senda, se plantean dos problemas fundamentales.

El primero, es que dada la conflictividad social del tema, la discusión técnica se obnubila y se pierde de vista al aumento de la productividad como fuente de competitividad El principal problema de las reformas laborales recientes es que no prestaron atención ni a la capacitación ni a ninguna otra fuente de aumento de la productividad, ni tuvieron en cuenta que la competitividad se define hoy día por la productividad.

El segundo es que un énfasis excesivo en el descenso de los costos laborales por hora obstaculiza el aumento de la productividad, generando así un escenario en el que lo que se alcanza en términos de competitividad vía descenso en los costos laborales por unidad. de trabajo, es parcial o totalmente neutralizado por lo que se pierde en términos de aumento de la productividad. Un ejemplo del tipo de obstáculo para el aumentó de la 
productividad generado por un énfasis excesivo en la reducción de los costos laborales por unidad de trabajo, es el efecto de la inestabilidad sobre la capacitación.

Chacaltana, J. y García, N. demuestran con evidencia empírica para el Perú, que existe estadísticamente una correlación negativa y significativa entre inestabilidad laboral y capacitación. Esto es, como cabría esperar, que una mayor inestabilidad laboral reduce la probabilidad de que las empresas gasten en capacitación de su personal, - y las presiona en la dirección de "robar" o contratar personal calificado por otras empresas. Los mismos autores estiman también empíricamente que en el segundo quinquenio de los 90 existía una elasticidad cercana a 0.10 entre el aumento de la productividad aparente a nivel micro - producto por ocupado en la empresa- y el gasto en capacitación por empresa. Esto es, que un aumento (reducción) de 10 por ciento en el gasto en capacitación por empresa, contribuía a elevar (contraer) en casi un 1 por ciento la productividad aparente.

La conclusión que se deriva del párrafo previo es muy importante: un cambio institucional - como el uso excesivo de contratos en negro y flexibles - produce un significativo aumento de la inestabilidad laboral que afecta negativamente el gasto medio en capacitación de las empresas, limitando así el aumento de la productividad.

En la practica, dado que no existían en el Perú recursos para establecer un sistema de capacitación que permitiera externalizar a las empresas los costos de formación de la mano de obra, el único ajuste restante era sincronizar el plazo requerido para tornar rentable una inversión de las empresas en capacitación, con el plazo prevaleciente de los contratos laborales, - lo que no significa igualar ambos plazos. Claramente, en una situación en la que el 80 por ciento de los asalariados se encuentran con contratos con una duración inferior a los dos años, y una porción muy grande de los mismos con contratos de una duración inferior al año, esto es un obstáculo porque las empresas requieren de un horizonte mucho más prolongado para que una inversión en capacitación sea rentable. Es prácticamente imposible esperar una rentabilidad de entre 60 y 120 por ciento en un plazo de uno a dos años, para la inversión en capacitación de las empresas, como sería lo requerido para recuperar la inversión inicial y tener una ganancia, en un plazo tan corto.

Por consiguiente, el cambio hacia múltiples contratos que reducen el plazo de la relación laboral, legitimado "ex ante" con la afirmación de que contribuye a mejorar la competitividad vía la reducción de los costos laborales por unidad de trabajo, en realidad afecta negativamente la productividad y el efecto neto sobre los costos laborales por unidad producida es empíricamente mucho menor o incierto. El cambio en la institucionalidad efectuado en el Perú contribuyó a reducir los costos laborales por unidad de trabajo, pero afectó negativamente a la productividad y con ello a los costos laborales por unidad producida.

El corolario de esta sección es que la institucionalidad laboral no debería obstaculizar ninguno de los dos caminos, ni la reducción de costos laborales por unidad de trabajo ni el aumento de la productividad. Por consiguiente, todas las reformas que efectuaron un énfasis desmedido en la multiplicidad de contratos para abaratar costos por unidad de 
trabajo y no prestaron atención alguna al aumento de la productividad, se tornan tarde o temprano en un obstáculo para la instalación de políticas deliberadas para el aumento de la productividad. Un país puède estar haciendo un esfuerzo muy grande en capacitación, acceso a innovaciones para la pequeña y mediana empresa, formación gerencial en gestión de la productividad, etc; pero si la institucionalidad laboral sigue orientada en la dirección de reducir los costos laborales por unidad de trabajo, es muy probable que el grueso de los empresarios pequeños y medianos, opten en la practica por el camino más fácil: la reducción de los costos laborales por unidad de trabajo, transformándose con ello en la principal restricción al aumento sistemático de la productividad.

\section{PROductividad Y COMPETITIVIDAD}

Para Sachs, J., Porter, M. y Warner, A., la competitividad está determinada por el nivel y crecimiento de la productividad. El nivel y crecimiento de la productividad son explicados por: i) el nivel tecnológico en un sentido amplio, incluyendo no sólo la capacidad de innovar y adaptar nuevas tecnologías sino el tipo de tecnología prevalecientes en los distintos segmentos y tamaños de empresas, y la forma de competir de las mismas; ii) el stock de capital en un sentido amplio, incluyendo el nivel educacional, la calificación y actitud gerencial, y el conjunto de prácticas e instituciones que de hecho $y$ de derecho regulan el comportamiento de las empresas.

Se ha enfatizado en negrillas en el párrafo previo dos factores muy relevantes para la experiencia Peruana y la mayor parte de las experiencias latinoamericanas. El primero, la importancia de que las empresas se habitúen a competir esencialmente por innovaciones, mejoras y aumento de la productividad, en lugar de competir sólo en base a costos de insumos y precios de productos. El segundo, la relevancia de la calificación y actitud gerencial y de las instituciones que de hecho y de derecho enmarcan el comportamiento de las empresas. El primero es decisivo y forma parte del desarrollo gradual de una "cultura de la productividad". El segundo es muy importante, y la institucionalidad del mercado laboral que incide sobre la conducta de las empresas es parte del mismo.

La productividad relevante es la productividad de todos los factores o recursos, y no sólo del insumo trabajo. El punto central es como reducir costos a través de mejoras sistemáticas de la productividad de todos los insumos y factores. No obstante, la productividad del trabajo es esencial en dos sentidos: por su importancia en sí misma y porque en muchas ocasiones, el aumento de la productividad de otros insumos. requiere también de una mejora simultánea en la productividad de la mano de obra : un nuevo equipo o una nueva organización interna serán más productivas en la mediàa que los trabajadores hayan sido entrenados previamente para las nuevas funciones.

Un aspecto esencial para la mayoría de los países latinoamericanos es que; salvo. 
contadas excepciones, la brecha de productividad entre los mismos y los países más desarrollados, ha tendido a ampliarse en las dos últimas décadas, a diferencia de lo ocurrido en el mismo período en muchos de los países del Asia. El Perú no ha sido una excepción a esta tendencia. Así, la diferencia en términos de productividad agregada e industrial entre EE.UU. y Perú era en el 2000 mucho mayor que la verificada en 1980. Por consiguiente, para países caracterizados por el atraso relativo en términos de innovaciones y productividad, la mejora de competitividad vía aumentos de la productividad es doblemente importante.

Un primer enfoque estratégico establece que con la política macroeconómica correcta y un marco de seguridad jurídica y credibilidad, el ajuste de los mercados conducirá espontáneamente a aumentos de la productividad. Uno de los aspectos más notables que emergen en los últimos dos decenios es el abandono del enfoque en el que la productividad es una variable exógena, determinada esencialmente por un ritmo exógeno de incorporación de nueva tecnología, y su reemplazo por la noción de que hoy en día, es factible desarrollar políticas a nivel de país, sector y empresas, orientadas a inducir y fortalecer el aụmento sistemático de la productividad a través de cambios organizacionales de todo tipo y la introducción de innovaciones de producto y de proceso.

Un segundo enfoque estratégico, basado en la premisa que la política macroeconómica no incide sobre mercados perfectamente competitivos sino sobre mercados imperfectos (Stiglitz, J., 2001 y 2002), plantea la necesidad de desarrollar políticas deliberadas y sobre todo un entorno institucional para las empresas, que induzca aumentos de productividad en plazos inferiores a los que el libre juego de los mercados pueda alcanzar, (García, N., 2002). Este segundo enfoque visualiza el aumento de la productividad como un área de política económica.

El punto esencial de este enfoque es sincronizar las políticas definidas a nivel macroeconómico que inciden sobre la productividad (acceso difundido a salud, acceso a importaciones de equipos, un sistema de formación laboral, mejoras del sistema educativo, promoción de exportaciones y ampliación de mercados externos, etc.) con un conjunto de cambios en las instituciones que sirvan de marco, moldeen y estimulen a nivel de la empresa la adopción de estrategias permanentes y sistemáticas hacia el aumento de la productividad (institucionalidad laboral, reglas del juego que influyen sobre el acceso a innovaciones o que inciden en la decisión de innovar y adoptar cambios; institucionalidad financiera; los canales institucionales existentes para difundir nuevos conocimientos entre las empresas; difusión generalizada de una capacidad gerencial a tono con la gestión de la productividad, y en general, la no existencia de reglas del juego que incidan en la otra dirección: obstaculizando la adopción de decisiones macroeconómicas de aumento de la productividad).

El enfoque de la competencia en base a productividad posee una implicancia no despreciable. En el enfoque de reducción de costos laborales a través de la adopción de múltiples contratos, puede esperarse un descenso de una sola vez de 20 o 30 por 
ciento de los costos laborales - lo que en el Perú implica un descenso de aproximadamente 8 a 10 por ciento en los costos totales -, en un período de cinco o diez años, en el que se enfrentará una fuerte conflictividad social que sin duda deja su huella y su herencia en una sociedad. Este enfoque tiene entonces un límite cuantitativamente evaluable, en cuanto a su aporte a un aumento de la competitividad y una exigencia en cuanto a aceptar y controlar la conflictividad que implica. El enfoque difundido del aumento de la productividad de todos los recursos, permite reducir los costos totales por unidad - y no sólo los laborales, en un 2 a 4 por ciento por año, durante decenios, y es más relevante aún para países emergentes en los que se verifica una significativa distancia con las tecnologías disponibles a nivel mundial: les queda mucho margen de adaptación de innovaciones para ser aprovechado. Simultáneamente el enfoque de la productividad permite mejoras de la competitividad sin que ellas sean obtenidas a expensas del bienestar y calidad de vida de un actor social. De hecho, es perfectamente compatible mejorar la rentabilidad de las empresas expuestas a la competitividad y también el nivel de bienestar de sus trabajadores, si los aumentos de productividad son permanentes y significativos.

Finalmente, conviene destacar la compatibilidad de una estrategia de competitividad. que descanse en aumentos sistemáticos de productividad, con el aumento del empleo.

En un modelo dinámico abierto de mediano plazo, el aumento de la productividad es perfectamente compatible e imprescindible para la generación de empleo de calidad, en la medida que contribuye a elevar la rentabilidad y a una mayor tasa de crecimiento de la inversión en transables y siempre que existan en operación políticas orientadas a ampliar la demanda externa a un ritmo bastante más alto que el aumento de la productividad (García, N., 2002). Un ejemplo numérico para el Perú contribuye a explicar el tema. El crecimiento esperado de la Población Económica Activa (PEA) urbana para el 2000- 2015 se encuentra cercano al 3.2 por ciento anual, lo que implica una meta de creación de empleo urbano superior al 4 por ciento anual para absorber el crecimiento anual de la PEA y reducir gradualmente el subempleo, el desempleo y la informalidad. Por el otro lado, la mejora en la competitividad demandará un crecimiento de la productividad aparente del orden del 3,5 por ciento anual para tener un impacto decisivo sobre costos totales unitarios. Los dos efectos suponen, al ser agregados, un crecimiente del PIB urbano cercano al 7.5 por ciento anual. Lo que implica un crecimiento del PIB agregado del orden del 6.5 por ciento anual. Pero este último, para materializarse, requiere un crecimiento de la inversión privada cercano al 7.5 por ciento anual y dadas las elasticidades importaciones/ producto prevalecientes, un crecimiento de las exportaciones superior al 8 por ciento anual para hacer viable dicho crecimiento. En consecuencia, el problema no es sólo acelerar el crecimiento económico, sino reorientar recursos y esfiuerzos hacia una mayor productividad en producción exportable y competitiva con importaciones:

En la práctica, esto exige que se encuentren activas :i) una política de tasa de cambio real apropiada para inducir producción exportable de bienes y servicios y produceión 
competitiva con importaciones; ii) una política de ampliación e incorporación de nuevos mercados externos, definida en acuerdo entre Gobierno y Sector Privado; iii) una política de nuevos acuerdos comerciales y financieros con países de mayor desarrollo relativo; iv) la negociación de accesos preferenciales a distintos mercados desarrollados.

En la práctica, esto implica elevar el crecimiento de las exportaciones y de la producción competitiva con importaciones, bastante por encima del aumento de la productividad. Supone un doble efecto por el lado de la demanda: i) el aumento inicial de la demanda génerado por la expansión de las exportaciones y la reducción del coeficiente de importaciones; ii) el aumento inducido de la demanda interna inducido por la expansión previa, al posibilitar un manejo más expansivo de la política económica. Este doble efecto por el lado de demanda, tiene su impacto en la aceleración del crecimiento económico y la creación de nuevos empleos de calidad. Por consiguiente, la óptica esencial no es introducir un conjunto de políticas al nivel macro, meso y micro para inducir un aumento sostenido de la productividad, sino hacerlo en forma consistente con la ampliación permanente de mercados externos y reducción del coeficiente de importaciones. De otro modo se afectaría aún más el empleo.

Como se desprende de lo expuesto, en forma paradojal, la mejor estrategia de empleo descansa en una mezcla balanceada de políticas orientadas a ampliar decisivamente mercados externos y reducir la propensión a importar, con políticas dirigidas a elevar sistemáticamente la productividad de todos los recursos, particularmente de la mano de obra. En una economía inserta en la globalización, es poco viable la generación de empleos de calidad que no esté sustentada en una clara competitividad de las empresas y del país, lo que implica alcanzar y sostener niveles aceptables de productividad. Pero también es cierto que se necesita generar muchos empleos de calidad, lo que implica una fuerte y permanente ampliación de mercados externos.

En esa perspectiva, el principal obstáculo es la ampliación de mercados externos al ritmo requerido por la ampliación de demanda para la producción transable doméstica. Las restricciones al comercio de los países más avanzados son hoy día el principal problema para acelerar el crecimiento económico, el de la productividad y el del empleo en los países emergentes. No es una casualidad que muchos de los esfuerzos de los países de América Latina se hayan orientado, por diferentes caminos, a lograr la ampliación de mercados externos. El principal desafío no está en las reformas internas - que en muchos países se encuentran ya en fases avanzadas - sino en el acceso a mercados de países - desarrollados y en la coherencia de las políticas domésticas para elevar la competitividad en base a productividad.

\section{5: CONCiusiones}

La evidencía empírica para el Perú sugiere que un exceso de flexibilidad de entrada al mercado laboral, vía múltiples contratos más "baratos" pero inestables, puede ser exitosa 
para reducir el costo laboral por unidad de trabajo, pero al mismo tiempo, constituirse en un obstáculo para la reducción del costo laboral por unidad producida - que es el indicador relevante para fines de competitividad. La mayor inestabilidad conspira contra el aumento de la productividad, que es el principal determinante de la competitividad -, y factor explicativo de los costos por unidad producida.

En esa perspectiva, una de las mayores contribuciones del mercado laboral a la mejora de la competitividad, es que tanto su institucionalidad como la conducta de empresas, gerentes, sindicatos y trabajadores, se desarrolle en forma pro activa respecto a un aumento sistemático de la productividad.

Este enfoque supone un impulso permanente hacia el aumento de la productividad de todos los recursos y factores. Es a través del mismo que es factible lograr descensos sistemáticos, en plazos prudenciales, en los costos totales por unidad de producto. Estos descensos son mucho más importantes para la competitividad, que los alcanzables a través de la reducción puntual de los costos laborales por unidad de trabajo.

Lo anterior no implica un enfoque opuesto a la flexibilidad, sino una ponderación más realista y un objetivo más adecuado para orientarla. La evidencia sugiere que un exceso de flexibilidad de entrada, es aún más perjudicial que la ausencia de la misma. Como en otros campos de la política económica, la dosificación de las medidas orientadas a modificar una realidad es imprescindible. En este sentido, el punto clave es no introducir más flexibilidad de entrada de la que sea necesaria para permitir la adaptación de las empresas a un entorno cambiante. Y sobre todo, impedir que se alcance una situación en que el exceso obstaculice el aumento de la productividad. La conclusión entonces es adoptar un referente empírico en base a un análisis del mercado laboral propio - en lugar de "importar" recetas y aplicarlas "a tabla rasa" - que permita establecer en qué rango debería moverse la proporción de asalariados con contratos no estables y adecuar la institucionalidad a este objetivo. Esto podrá significar en algunas experiencias un 10015 por ciento de los asalariados sujetos a contratos no estables y en otras un 20 o 30 por ciento. Pero en ningún caso un 80 por ciento como el alcanzado en el Perú a principios de la presente década.

Para los países que carecen de un sistema eficaz de formación de la mano de obra, una referencia adicional es impedir que los plazos predominantes en materia de contratos laborales, se reduzcan muy por debajo del plazo requerido para hacer rentable la inversión de las empresas en capacitación. Un exceso de contratos no estables conduce a este hecho, y frena cualquier iniciativa privada para ampliar la capacitación de los trabajadores.

La segunda conclusión es que para compatibilizar aumentos de productividad con incrementos en el empleo de calidad, es imprescindible concentrar esfuerzos asociados del Gobierno y del Sector Privado, para ampliar sistemáticamente mercados externos y reducir la proporción de importaciones. El ritmo de expansión de la producción exportable y competitiva con importaciones, determinará en la práctica la aceleración del crecimiento económico que se alcance, y con ello, el margen para elevar la productividad y el empleo. 
La tercera conclusión es que una estrategia de aumento de la productividad, requerirá una institucionalidad adecuada. En este sentido, será imprescindible desarrollar ámbitos de concertación a nivel micro, meso y macro, para desarrollar acciones concertadas entre Actores Sociales y Gobierno orientadas a promover las medidas necesarias para inducir un enfoque de aumento de la productividad y comprometer acciones en esta dirección. La opción de una estrategia de aumentos de la productividad para elevar la competitividad, implica en sí una alternativa menos conflictiva, socialmente, que la fuerte reducción de costos laborales en base a al aumento de la inestabilidad. Pero es muy importante establecer diversos ámbitos de negociación en los que esta estrategia pueda apoyarse.

Ia cuarta conclusión, se refiere a cual debería ser el foco de la flexibilidad. En este trabajo, por su importancia e impacto en la experiencia del Perú en los 90's, el análisis se centró en la flexibilidad de entrada. Pero existen en la práctica diferentes tipos de flexibilidad. El énfasis de este trabajo en la necesidad de elevar la productividad de todos los recursos para reducir los costos totales por unidad de producto y mejorar la competitividad, conduce a priorizar la flexibilidad para elevar la productividad de todos los recursos, en la empresa. Esto es, generar en el ámbito laboral una institucionalidad de hecho y de derecho que facilite y estimule la adopción de estrategias de aumento de la productividad a nivel de empresa. Esto supone un análisis un poco más complejo respecto a como incide la institucionalidad sobre estas decisiones, pero definitivamente más apropiado para los verdaderos desafíos que enfrentan buena parte de los países latinoamericanos para el futuro.

\section{Bibliografia}

Chacaltana, J. y García, N. (2001): Reforma Laboral, Capacitación y Productividad. OIT, Oficina Regional para América Latina y el Caribe, Lima.

García, N. (2002): Productividad, Competitividad y Empleo: Un Enfoque Estratégico. CIES, Preliminar, Lima.

García, N. (1999): Costos Laborales y Competitividad en el Perí. SISMEL/INEI, Lima. Heckman, J. (1999): Policies to foster buman capital. NBER Working Paper $\mathrm{N}^{\circ}$ 7288, Cambridge, Mass.

Ichniowski,C. Shaw, K. Prennushi, G. (1995): The effects of buman resource management practices on productivity. NBER Working Paper $\mathrm{N}^{\circ} \mathrm{W} 5333$, Cambridge, Mass. 1995

Martinez,D.y Tokman,V. (2001): Costos Laborales y Competitividad. OIT, Lima.

Porter, M. (1988): The competitive advantage of nations. The Free Press, New York. Sachs, J., Porter, M. y Warner, A. (2000): The Global Competitiveness Report 2000. Oxford University Press, New York, USA.

Stiglitz, J. (2001): The role of the State, New York, S.U.P..

Stiglitz, J.. (2002): El malestar en la globalización. Colección Taurus, Madrid. 